\title{
Preparation and Microstructural, Structural, Optical and Electro-Optical Properties of La Doped PMN-PT Transparent Ceramics
}

\author{
Fernando Andrés Londono Badillo, Jose Antonio Eiras, Flavio Paulo Milton, Ducinei Garcia \\ Ferroelectric Ceramics Group, Physics Department, Federal University of São Carlos, São Carlos, Brazil \\ Email: flondono@df.ufscar.br
}

Received June 30, 2012; revised July 27, 2012; accepted August 10, 2012

\begin{abstract}
Transparent relaxor ferroelectric ceramics of the system lanthanum modified lead magnesium niobate have been investigated for a variety of electro-optic properties that could make these materials alternatives to $(\mathrm{Pb}, \mathrm{La})(\mathrm{Zr}, \mathrm{Ti}) \mathrm{O}_{3}$. However, a study that relates the to properties in function stoichiometric formula, has not been analyzed heretofore. Therefore, in this work the effect of A-site substitution of $\mathrm{La}^{3+}$ in the characterization microstructural, structural, optical and electro-optical on $(1-x)\left[\mathrm{Pb}_{(1-3 / 2 \mathrm{y})} \mathrm{La}_{\mathrm{y}}\left(\mathrm{Mg}_{1 / 3} \mathrm{Nb}_{2 / 3}\right) \mathrm{O}_{3}\right]-x \mathrm{PbTiO}_{3}$ and $(1-z)\left[(1-x) \mathrm{Pb}\left(\mathrm{Mg}_{1 / 3} \mathrm{Nb}_{2 / 3}\right) \mathrm{O}_{3}+x \mathrm{PbTiO}_{3}\right]+z \mathrm{La}_{2} \mathrm{O}_{3}$ has been performed. It was observed that the properties according to the stoichiometric formula and the PT had a maximum whose behavior was related to the addition of lanthanum in each stoichiometries.
\end{abstract}

Keywords: Ferroelectric; Optics; Transmittance; Electro-Optical

\section{Introduction}

Lead magnesium niobato $\mathrm{Pb}\left(\mathrm{Mg}_{1 / 3} \mathrm{Nb}_{2 / 3}\right) \mathrm{O}_{3}$ ( $\mathrm{PMN}$ ), as one of the most widely investigated relaxor ferroelectric with a perovskites structure, was first synthesized in the late 1950s [1]. Initially, PMN was prepared by the conventional mixed oxide method, where pyrochlore phase was inevitably produced. In order to synthesize stoichiometric perovskite PMN ceramics, Swartz and Shrout [2] proposed a columbite precursor method, where the intermediate reaction of the formation of pyrochlore phase was bypassed, which results in the stabilization of perovskites structure as compared to the mixed oxide method. Recently, a novel methodology was devised to stabilize perovskites structure by adding stable normal $\mathrm{PbTiO}_{3}$ (PT). The formation of the solid solution increases the tolerance factor and electronegativity difference, leading to the stabilization of the perovskites structure and the enhancement of dielectric property of the relaxor ferroelectric [3].

A study of the optical, electrical and electro-optical properties of PMN-PT ceramics in function of the stoichiometrie is of interest both for possible insight into the physical nature of relaxor ferroelectrics as well as for making practical extension of its several present applications to include usage in electro-optical devices. Present applications take advantages of PMN-PT singularly ex- cellent dielectric, low thermal expansion, and high electrostrictive properties [4]. From the viewpoint of crystal chemistry, the substitution of $\mathrm{Ti}^{4+}$ ions for the complex $\left(\mathrm{Mg}_{1 / 3} \mathrm{Nb}_{2 / 3}\right)^{4+}$ ions on the B-site of the perovskite structure in the $(1-x) \mathrm{Pb}\left(\mathrm{Mg}_{1 / 3} \mathrm{Nb}_{2 / 3}\right) \mathrm{O}_{3}-x \mathrm{PbTiO}_{3}$ (PMNPT) system leads to the outstanding properties of the PMN-PT ceramics that exhibit excellent electrical and electro-optical performance, which make them promising applications in multilayer capacitors, piezoelectric transducers and actuators and optical devices $[5,6]$. However, perovskite structure are extremely difficult to fabricate reproducibly without the appearance of stable pyrochlore phase, which always exist in the PMN-PT ceramics and significantly deteriorates such properties as the dielectric property [7].

The optical transmittance as function of wavelength was studied in $\mathrm{Pb}\left(\mathrm{Mg}_{1 / 3} \mathrm{Nb}_{2 / 3}\right)_{0.62} \mathrm{Ti}_{0.38} \mathrm{O}_{3}$ single crystal by Wan et al. It was found that the crystal is transparent in the visible region and rolls off in near $450 \mathrm{~nm}$. Using the Senarmont compensador method, the effective electro-optic coefficient $r_{c}=42.8 \mathrm{pm} / \mathrm{V}$ was also obtained [8]. Some effect and applications, such as photorefractive, Second-Harmonic Generation (SHG), electro-optic and elasto-optical devices, are based on the large optical property coefficient of the materials $[9,10]$. The knowledge of factors that can modify these properties is desirable to find new application of the relaxor and elec- 
tro-optical materials. In this work, ceramics in the PMN-PT system were prepared by doping with lanthanum. La addition to PMN-PT has been shown to promote densification and through hot uniaxial pressing, optically transparent materials have been achieved, allowing the determination of various optic and electro optic properties [11,12].

It is the purpose of this article, to report the microstructural, structural, electrical, optical and electro-optical properties according to the stoichiometric formula of lanthanum doped PLMN-XPT and PMN-XPT:La with $0.11 \leq$ $\mathrm{x} \leq 0.15$.

\section{Experimental Procedure}

The powder was synthesized by the columbite or twostage calcining method [2]. The batch formulae were $(1-x)\left[\mathrm{Pb}_{(1-\mathrm{y})} \mathrm{La}_{\mathrm{y}}\left(\mathrm{Mg}_{1 / 3} \mathrm{Nb}_{2 / 3}\right) \mathrm{O}_{3}\right]-x \mathrm{PbTiO}_{3}$

PLMN-XPT, and

$(1-z)\left[(1-x) \mathrm{PbLa}\left(\mathrm{Mg}_{1 / 3} \mathrm{Nb}_{2 / 3}\right) \mathrm{O}_{3}-x \mathrm{PbTiO}_{3}\right]+z \mathrm{LaO}_{3 / 2}$ PMN-XPT:La, with $y=0.01 . z=0.01, \mathrm{X}=100 x$ and $0.11 \leq x \leq 0.15$. The starting materials were lanthanum oxide, $\mathrm{La}_{2} \mathrm{O}_{3}$ (Aldrich, $>99 \%$ purity), niobium oxide, $\mathrm{Nb}_{2} \mathrm{O}_{5}$ (Alfa Aesar 99.9\% purity), magnesium carbonate hydroxide pentahydrate, $\left(\mathrm{MgCO}_{3}\right) 4 \cdot \mathrm{Mg}(\mathrm{OH})_{2} \cdot 5 \mathrm{H}_{2} \mathrm{O}(\mathrm{Al}-$ drich $99 \%$ purity), lead oxide, $\mathrm{PbO}$ (MGK 99\%) and titanium oxide, $\mathrm{TiO}_{2}$ (Alfa Aesar, $99.8 \%$ purity) powders. The $\left(\mathrm{MgCO}_{3}\right) 4 \cdot \mathrm{Mg}(\mathrm{OH})_{2} \cdot 5 \mathrm{H}_{2} \mathrm{O}$ was carried up to $1100^{\circ} \mathrm{C}$, for $4 \mathrm{~h}$, to drive off $\mathrm{CO}_{2}$ and $\mathrm{H}_{2} \mathrm{O}$ and obtain the correct amount of $\mathrm{MgO}$ for a stoichiometric reaction with $\mathrm{Nb}_{2} \mathrm{O}_{5}$. In the first stage, $\mathrm{MgO}$ and $\mathrm{Nb}_{2} \mathrm{O}_{5}$ powders were ballmilled and prereacted at $1100^{\circ} \mathrm{C}$ for $4 \mathrm{~h}$, in air, to form the columbite phase $\left(\mathrm{MgNb}_{2} \mathrm{O}_{6}\right)$. In the second stage, the synthesized $\mathrm{MgNb}_{2} \mathrm{O}_{6}(\mathrm{MN})$ was ball-milled in isopropanol, for $24 \mathrm{~h}$, with appropriate amounts of $\mathrm{PbO}, \mathrm{TiO}_{2}$ and $\mathrm{La}_{2} \mathrm{O}_{3}$ and heated at $900^{\circ} \mathrm{C}$, for $4 \mathrm{~h}$, in oxygen atmosphere, at a controlled pressure of $200 \mathrm{kPa}$. The calcined powders were pressed into pellets $10 \mathrm{~mm}$ in diameter and $10 \mathrm{~mm}$ in thickness with the addition of an appropriate amount of polyvinyl alcohol (PVA) binder. The pellets were sintered in hot uniaxial pressing in $\mathrm{O}_{2}$ atmosphere followed for $4 \mathrm{~h}$ at $1220^{\circ} \mathrm{C}$, and $6 \mathrm{MPa}$.

The phases and the structural parameters were determined by X-ray diffraction (XRD) using a Rigaku diffractometer, with $\mathrm{CuK} \alpha$ radiation. The lattice parameters were calculated by least squares from the positions of the diffraction peaks. Apparent densities were determined by the Archimedes method. The microstructural features of the samples were investigated by scanning electron microscopy (SEM), using a Jeol JSM 5800 LV. The grain sizes were calculated from the SEM images of the polished thermally etched surfaces by the linear-intercept method. For dielectric measurement, gold electrodes were deposited on both faces of the disk samples $(5 \mathrm{~mm}$ diameter and $\sim 1.0 \mathrm{~mm}$ thick). The relative permittivity, $\varepsilon$ was measured by impedance analysis (HP4194A). The transmittance was measured in a spectrophotometer (Micronal-B582), at wavelengths ranging from 200 to 1000 $\mathrm{nm}$ for optically polished samples, $600 \mu \mathrm{m}$ thick. The method used in the electro-optical characterization, determination of the Pockels and Kerr coefficients, is based in the Senarmont configuration illustrated in the Figure 1 [13]. The birefringence medium (sample) is located between two linear polarizer's, $\mathrm{P}_{1}$ and $\mathrm{P}_{2}$, where the $\mathrm{P}_{2}$ polarizer is known as analyzer, whit polarizer axis forming angles of $\pm 45^{\circ}$ in relation to principal axis of the sample, this definite by the electric-field direction applied. Between the sample and the analyzer is located a $\lambda / 4$ plate used to compensation the effects caused by the natural birefringence of the medium. Then the analyzer is positioned in the angle $\beta$ that optimizes the relation between the birefringence and the applied electric-field. In the Equation (1) is presented the relation between birefringence and electric-field for medium with linear and quadratic electro-optic response.

$$
\Delta n(E)=-1 / 2 n^{3}(r E+R E)
$$

where $\Delta n$ is the birefringence induced for the electric-field $E, n$ is the natural birefringence of the medium, $r$ and $R$ are the Pockels and Kerr electro-optic coefficients, respectively.

\section{Results and Discussion}

The relative density of PLMN-XPT and PMN-XPT:La at different PT concentration are greater than $96 \%$ of the theoretical density as can be seen in Figure 2, which is especially suitable for electronic industry application [14]. The relative density of the two system exhibits the tendency to decrease when PT concentration is increased. This can be attributed the loss of $\mathrm{PbO}$ (control atmosphere of $\mathrm{PbO}$ was not used in this work) [15]. As well can be seen the relative densities of PLMN-XPT ceramics is greater for all PT concentrations when compared with PMN-XPT:La. This possibly associated with the less occupation of $\mathrm{La}^{3+}$ in $\mathrm{Pb}^{2+}$ sites, implying in the appearance of vacancies in the $\mathrm{A}$ and $\mathrm{B}$ sites in different quantities [16] $\left(\mathrm{La}^{3+}\right.$ atoms are lighter than $\mathrm{Pb}^{2+}$ atoms, and PMN-XPT:La have major number of $\mathrm{La}$ atoms).

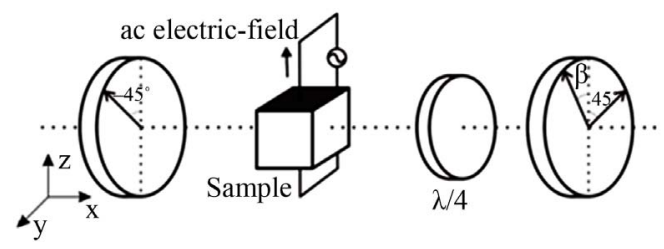

Figure 1. Illustration of the experimental Senarmont method for characterization of the transversal electro-optical effect. 


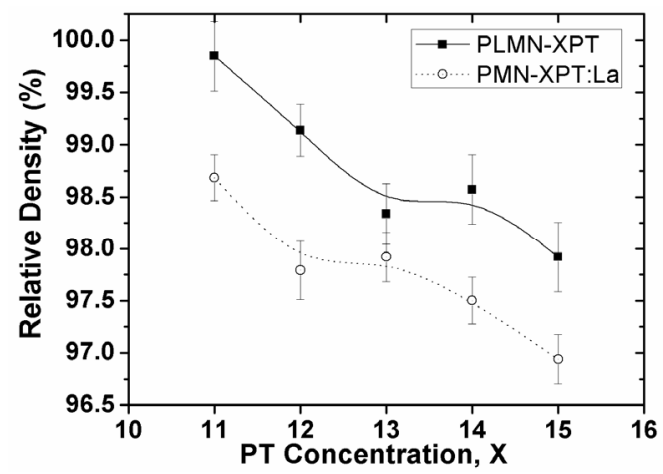

Figure 2. Variation of relative density as a function of PT concentration of the PLMN-XPT and PMN-XPT:La ceramics with $0.11 \leq x \leq 0.15$.

The variation of density is considered as relating to mass loss induced by evaporation of $\mathrm{PbO}$ or to variation of temperature of densification relating to the increase of PT, this changes the grain size, porosity could appear which decreases the relative density of ceramics. However the slight decrease of relative density of PLMN-XPT and PMN-XPT:La ceramics can be attributed to the variation of microstructure and to evaporation of lead at elevated sintering temperatures.

The high densification of PLMN-XPT and PMN-XPT:La ceramics is further confirmed by SEM observation, which is shown in Figure 3. All ceramics exhibited similar microstructure as can be seen in the micrograph of polished and thermally attacked surface of PLMN-13PT (shown here).

The variation of grain size with the increase of PT concentration is shown in Figure 4. An increase in PT concentration resulted in a decrease in grain size and a corresponding slight decrease in density [17]. The grain size in PMN-XPT:La is lesser for all concentration when compared to PLMN-XPT because the major quantities of $\mathrm{La}^{3+}$ resulted in reduction of grain size as observed by Kim et al. in $\mathrm{La}^{3+}$ doped PMN-PT ceramics [18].

The XRD patterns of sintered ceramics with different compositions are presented in Figure 5, where a complete perovskite structure (JCPDS 391488) is formed and the pyrochlore or other second phases were not detected.

The permittivity (real, $\varepsilon^{\prime}$ and imaginary, $\varepsilon^{\prime \prime}$ ) was measured at various temperatures in the frequency range 1 $\mathrm{kHz}-1 \mathrm{MHz}$. In the Figure 6 can be seen a typical examples of measurements realized in this work. All the samples exhibit typical relaxor behavior [19,20], with the magnitude of $\varepsilon^{\prime}$ decreasing with increasing frequency, and the maximum permittivity real, $\varepsilon_{\text {max }}^{\prime}$ shifting to higher temperatures, as shown in the PLMN-12PT ceramics (Figure 6). The data of all ceramic analyzed in this work can be seen in the Table $\mathbf{1}$.

Variations in the shape of $\varepsilon^{\prime}$ in function of $T$ curves can be also directly related to the diffuseness coefficient,

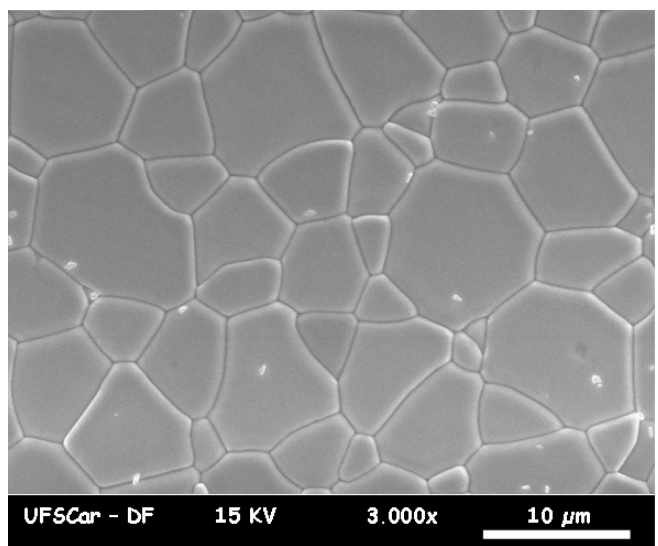

Figure 3. SEM image of surfaces polished and thermally attacked of PLMN-13PT ceramic.

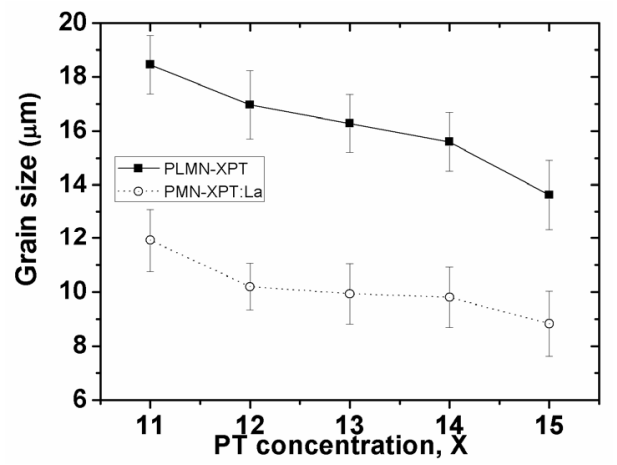

Figure 4. Grain size in function of $P T$ concentration of the PLMN-XPT and PMN-XPT:La ceramics with $0.11 \leq x \leq 0.15$.
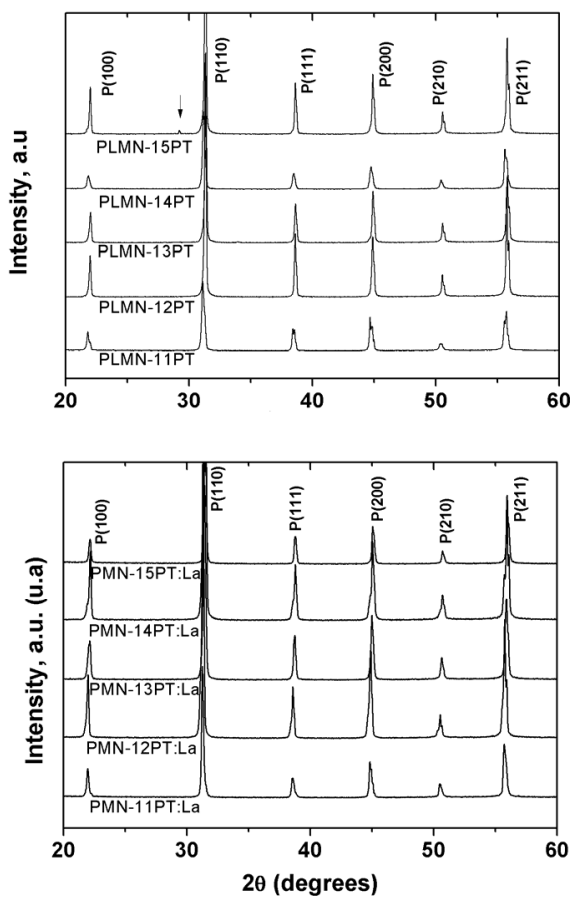

Figure 5. XRD patterns of PLMN-XPT and PMN-XPT:La ceramics with $0.11 \leq x \leq 0.15$. 


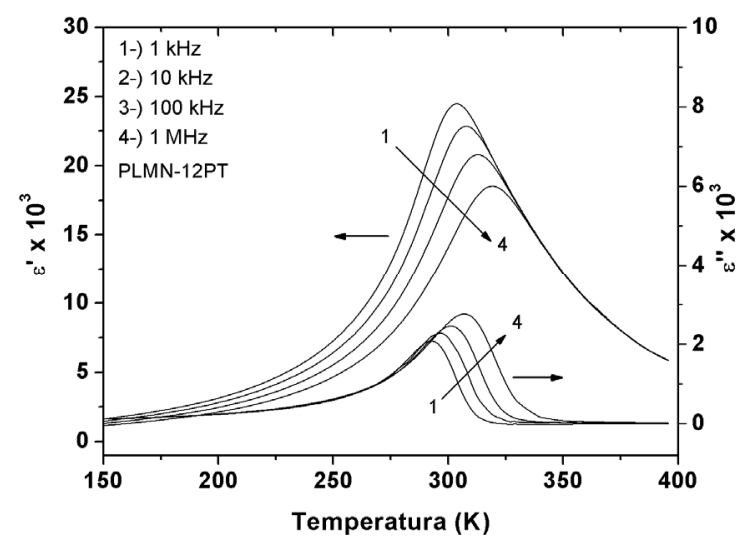

Figure 6. Permittivity real, $\varepsilon^{\prime}$ and permittivity imaginary, $\varepsilon^{\prime \prime}$ at various frequencies in function of temperature for PLMN-XPT and PMN-XPT:La ceramics with $0.11 \leq x \leq 0.15$.

Table 1. Dielectric properties of PLMN-XPT and PMN-XPT: La with $0.11 \leq x \leq 0.15$ at $1 \mathrm{kHz}$.

\begin{tabular}{cccc}
\hline Ceramic & $\varepsilon_{\text {max }}^{\prime}$ & $\mathrm{T}_{\max }(\mathrm{K})$ & $\Delta$ \\
\hline PLMN-11PT & 27,000 & 293 & 1.66 \\
PLMN-12PT & 26,800 & 300 & 1.65 \\
PLMN-13PT & 25,200 & 305 & 1.63 \\
PLMN-14PT & 29,000 & 308 & 1.53 \\
PLMN-15PT & 28,000 & 317 & 1.55 \\
PMN-11PT:La & 25,000 & 297 & 1.62 \\
PMN-12PT:La & 24,700 & 299 & 1.62 \\
PMN-13PT:La & 25,100 & 308 & 1.62 \\
PMN-14PT:La & 23,000 & 318 & 1.60 \\
PMN-15PT:La & 30,000 & 319 & 1.52 \\
\hline
\end{tabular}

$\delta$ in Table 1, calculated from the Santos Eiras equation [21], which for relaxors reflect the level of disorder in term of the diffusivity of the $\varepsilon_{\text {max }}^{\prime}$. As can be deduced from Table 1, the value of $\varepsilon_{\text {max }}^{\prime}$ for PMN-XPT: $\mathrm{La}^{3+}$ are smaller that PLMN-XPT, inferring that lanthanum additions result in the reduction in $\varepsilon_{\text {max }}^{\prime}$, while increasing the degree of ordering, as observed for Kim et al. [18].

The transmission spectrum as a function of wavelength (200 nm - $1100 \mathrm{~nm}$ ) of PLMN-XPT and PMN-XPT:La ceramics with $0.11 \leq x \leq 0.15$ and $630 \mu \mathrm{m}$ thickness is presented in Figure 7. For both of these stoichiometries formula the percentage of transmitted light begins to rise abruptly at just below $380 \mathrm{~nm}$ and then increases only gradually with wavelength about $500 \mathrm{~nm}$. This gradual increase in transmittance continues into the near IR at least through $1100 \mathrm{~nm}$ without any noticeable absorption band being observed. This is similar to what was observed for most crystals with oxygen-octahedral perovskites structure [22-24]. From the transmission characterization, we can see that the optical absorption is very small at higher wavelength range. In general, optical transmission relates to reflection loss and scattering loss. Using the refractive indices measured by Mchenry et al. [12], e by the Fresnel expression, the reflection loss of the light at two surfaces was calculated about $20 \%$. Beyond of reflection loss is observed loss by scattering which can be attributed the dispersion by ferroelectrics domain (spontaneous birefringence), increases in the porosity (by loss of $\mathrm{PbO}$ ) and precipitation of spurious phases. In general the addition of PT increase the transmittance both the PLMN-XPT and PMN-XPT:La, nevertheless exist a concentration optimal of PT in $14 \%$. As previously mentioned the increase of content of PT promotes the densification of the samples, favoring the transmittance, though the sample with PMN-XPT:La present less transmission in comparison with the PLMN-XPT in function of wavelength. This effect is attributed the replacement of lanthanum ions in the sites of the lead, that increased the number of vacancies reducing the density and consequently affecting the transmittance.

In the Table 2 can be seen the values for the electrooptic Kerr coefficients as a function of PT concentration and stoichiometric formula, which were determined under frequency of $200 \mathrm{~Hz}$, at room temperature. The values of electro-optical coefficients here obtained are similar the reported in literature for La doped PMN-PT ce-
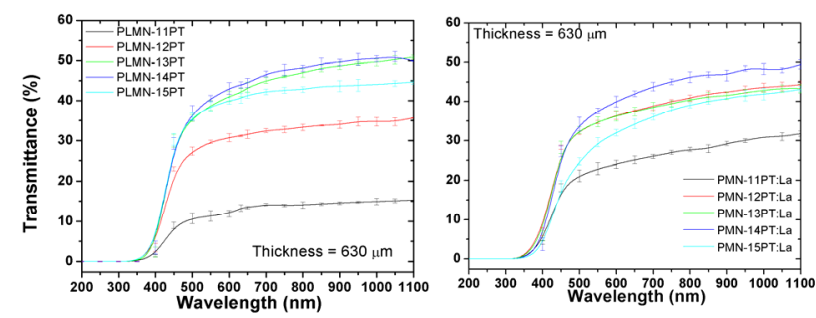

Figure 7. Percent transmission as a function of wavelength for optically polished PLMN-XPT and PMN-XPT:La with $0.11 \leq x \leq 0.15$ and $630 \mu \mathrm{m}$ thickness.

Table 2. Electro-optical coefficient, $R$ of PLMN-XPT and PMN-XPT:La with $0.11 \leq x \leq 0.15$ calculated under frequency of $200 \mathrm{~Hz}$.

\begin{tabular}{cc}
\hline Ceramic & $\mathrm{R}\left(\times 10^{-16} \mathrm{~m}^{2} / \mathrm{V}^{2}\right)$ \\
\hline PLMN-11PT & 8.0 \\
PLMN-12PT & 14.1 \\
PLMN-13PT & 23.7 \\
PLMN-14PT & 10.1 \\
PLMN-15PT & 10.3 \\
PMN-11PT:La & 7.1 \\
PMN-12PT:La & 10.3 \\
PMN-13PT:La & 13.0 \\
PMN-14PT:La & 9.2 \\
PMN-15PT:La & 6.1 \\
\hline
\end{tabular}


ramics $[12,25]$ and greater to commercial PLZT ceramics [26]. The Kerr coefficients of the PLMN-XPT ceramics are higher when compared with PMN-XPT:La ceramics because of their major transmittance, since that the phase induced by electro-optical effect is directly proportional intensity of light transmission [27].

\section{Conclusion}

The ceramics obtained in this study showed high dense and homogeneous microstructure, independent of the stoichiometric which were obtained. The La addition to the PLMN-XPT and PMN-XPT:La relaxor ferroelectric system was found to promote densification and inhibit grain growth, however the PMN-XPT:La ceramic to present smaller density in relation to other composition. The transmittance characterization revealed a transmission of about $50 \%$ from $0.45 \mu \mathrm{m}$ to $1.1 \mu \mathrm{m}$, presenting a lower transmission in the samples of PMN-XPT:La. The quadratic electro-optic coefficients, calculated from measurements of birefringence in this work to PMN-XPT and PLMN-XPT ceramics are up to two times higher than the maximum reported for PLZT. Thus confirming the potential use of these system as substitutes for PLZT in electro-optical applications.

\section{Acknowledgements}

To CAPES, FAPESP and CNPq for the financial support. To Dr. Y. P. Mascarenhas (São Carlos Physics Institute at the University São Paulo), for the use of the XRD laboratory facilities. And to Mr. Francisco José Picon for the technical support.

\section{REFERENCES}

[1] G. A. Smolenskii and A. I. Agranovskaya, "Dielectric Polarization and Losses of Some Complex Compounds," Soviet Physics-Technical Physics, Vol. 3, No. 7, 1958, pp. 1380-1382.

[2] S. L. Swartz and T. R. Shrout, "Fabrication of Perovskite Lead Magnesium Niobate," Materials Research Bulletin, Vol. 17, No. 10, 1982, pp. 1245-1250. doi:10.1016/0025-5408(82)90159-3

[3] T. R. Shrout and A. Halliyal, "Preparation of Lead-Based Ferroelectric Relaxors for Capacitors," The Bulletin of the American Ceramic Society, Vol. 66, No. 4, 1987, pp. 704711.

[4] S. J. Jang, K. Uchino, S. Nomura and L. E. Cross, "Electrostrictive Behavior of Lead Magnesium Niobate Based Ceramic Dielectrics," Ferroelectrics, Vol. 27, No. 1, 1980, pp. 31-34. doi:10.1080/00150198008226059

[5] Y. H. Chen, K. Uchino, M. Shen and D. Viehland, "Substituent Effects on the Mechanical Quality Factor of $\mathrm{Pb}\left(\mathrm{Mg}_{1 / 3} \mathrm{Nb}_{2 / 3}\right) \mathrm{O}_{3}-\mathrm{PbTiO}_{3}$ and $\mathrm{Pb}\left(\mathrm{Sc}_{1 / 2} \mathrm{Nb}_{1 / 2}\right) \mathrm{O}_{3}-\mathrm{PbTiO}_{3}$ Ceramics," Journal of Applied Physics, Vol. 90, No. 3, 2001, pp. 1455-1458. doi:10.1063/1.1379248
[6] L. B. Kong, J. Ma, W. Zhu and O. K. Tan, "Rapid Formation of Lead Magnesium Niobate-Based Ferroelectric Ceramics via a High-Energy Ball Milling Process," Materials Research Bulletin, Vol. 37, No. 3, 2002, pp. 459465. doi:10.1016/S0025-5408(01)00823-6

[7] E. M. Jayasingh, K. Prabhakaran, R. Sooraj, C. Durgaprasad and S. C. Sharma, "Synthesis of Pyrochlore Free PMN-PT Powder by Partial Oxalate Process Route," Ceramics International, Vol. 35, No. 2, 2009, pp. 591-596. doi:10.1016/j.ceramint.2008.01.022

[8] X. M. Wan, H. Q. Xu, T. H. He, D. Lin and H. S. Luo, "Optical Properties of Tetragonal $\mathrm{Pb}\left(\mathrm{Mg}_{1 / 3} \mathrm{Nb}_{2 / 3}\right)_{0.62-}$ $\mathrm{TiO}_{0.38}$ Single Crystal," Journal of Applied Physics, Vol. 93, No. 8, 2003, pp. 4766-4768. doi:10.1063/1.1561991

[9] A. S. Bhalla, R. Guo, A. S. Cross, G. Burns, F. H. Dacol and R. R. Neurgaonkar, "Glassy Polarization in the Ferroelectric Tungsten Bronze (Ba, $\mathrm{Sr}) \mathrm{Nb}_{2} \mathrm{O}_{6}$," Journal of Applied Physics, Vol. 71, No. 11, 1992, pp. 5591-5595. doi:10.1063/1.350537

[10] A. S. Bhalla, R. Guo, A. S. Cross, G. Burns, F. H. Dacol and R. R. Neurgaonkar, "Measurements of Strain and the Optical Indices in the Ferroelectric $\mathrm{Ba}_{0.4} \mathrm{Sr}_{0.6} \mathrm{Nbz}$ : Polarization Effects," Physical Review B, Vol. 36, No. 4, 1987, pp. 2030-2035. doi:10.1103/PhysRevB.36.2030

[11] F. A. Londono, J. A. Eiras and D. Garcia, "New Transparent Ferroelectric Ceramics with High Electro-Optical Coefficients: PLMN-PT," Cerâmica, Vol. 57, No. 344 2011, pp. 404-408.

[12] D. A. Mchenry, J. Giniewicz, S. J. Jang, A. Bhalla and T. R. Shrout, "Optical Properties of Hot Pressed Relaxor Ferroelectrics," Ferroelectrics, Vol. 93, No. 1, 1989, pp. 351-359. doi:10.1080/00150198908017367

[13] R. Go, "Ferroelectric Properties of Lead Barium Niobate Compositions near the Mosphotropic Phase Boundary," Ph.D. Theses, Pennsylvania State University, Pennsylvania, 1990.

[14] C. Ding, et al., "Phase Structure and Electrical Properties of $0.8 \mathrm{~Pb}\left(\mathrm{Mg}_{1 / 3} \mathrm{Nb}_{2 / 3}\right) \mathrm{O}_{3}$ Relaxor Ferroelectric Ceramics Prepared by the Reaction-Sintering Method," Physica Status Solidi (A), Vol. 207, No. 4, 2010, pp. 979-985. doi:10.1002/pssa.200925377

[15] G. S. Snow, "Fabrication of Transparent Electrooptic PLZT Ceramics by Atmosphere Sintering," Journal of the American Ceramic Society, Vol. 56, No. 2, 1973, pp. 9196. doi:10.1111/j.1151-2916.1973.tb12365.x

[16] S. M. Gupta and D. Viehland, "Role of Charge Compensation Mechanism in La-Modified $\mathrm{Pb}\left(\mathrm{Mg}_{1 / 3} \mathrm{Nb}_{2 / 3}\right) \mathrm{O}_{3^{-}}$ $\mathrm{PbTiO}_{3}$ Ceramics: Enhanced Ordering and Pyrochlore Formation," Journal of Applied Physics, Vol. 80, No. 10, 1996, pp. 5875-5883. doi:10.1063/1.363581

[17] N. Kim, D. A. McHenry, S. J. Jang and T. R. Shrout, "Fabrication of Optically Transparent Lanthanum Modified $\mathrm{Pb}\left(\mathrm{Mg}_{1 / 3} \mathrm{Nb}_{2 / 3}\right) \mathrm{O}_{3}$ Using Hot Isostatic Pressing," Journal of the American Ceramic Society, Vol. 73, No. 4, 1990, pp. 923-928. doi:10.1111/j.1151-2916.1990.tb05137.x

[18] N. Kim, W. Huebner, S. J. Jang and T. R. Shrout, "Dielectric and Piezoelectric Properties of Lanthanum-Modified Lead Magnesium Niobium-Lead Titanate Ceram- 
ics," Ferroelectrics, Vol. 93, No. 1, 1989, pp. 341-349. doi:10.1080/00150198908017366

[19] L. E. Cross, S. J. Jang, R. E. Newnham, S. Nomura and K. Uchino, "Large Electrostrictive Effects in Relaxor Ferroelectrics," Ferroelectrics, Vol. 23, No. 1, 1980, pp. 187191. doi:10.1080/00150198008018801

[20] K. Uchino, S. Nomura, L. E. Cross, S. J. Jang and R. E. Newnham, "Electrostrictive Effect in Lead Magnesium Niobate Single Crystals," Journal of Applied Physics, Vol. 51, No. 2, 1979, pp. 1142-1145. doi:10.1063/1.327724

[21] I. A. Santos and J. A. Eiras, "Phenomenological Description of the Diffuse Phase Transitiion Inferroelectrics," Journal of Physics: Condensed Matter, Vol. 13, No. 50, 2001, pp. 11733-11740. doi:10.1088/0953-8984/13/50/333

[22] X. M. Wan, H. S. Luo and X. Y. Zhao, "Refractive Indices and Linear Electro-Optic Properties of $(1-x) \mathrm{Pb}\left(\mathrm{Mg}_{1 / 3} \mathrm{Nb}_{2 / 3}\right) \mathrm{O}_{3}-x \mathrm{PbTiO}_{3}$ Single Crystals," $A p$ plied Physics Letters, Vol. 85, No. 22, 2004, pp. 52335235. doi:10.1063/1.1829393

[23] C. J. He, L. H. Luo and X. Y. Zhao, “Optical Properties of Tetragonal Ferroelectric Single Crystal Lead Magnesium Niobate Lead Titanate," Journal of Applied Physics, Vol. 100, No. 1, 2006, Article ID: 013112. doi:10.1063/1.2217487

[24] F. A. Londono, J. Eiras and D. Garcia, "Optical and Electro-Optical Properties of $(\mathrm{Pb}, \mathrm{La}) \mathrm{TiO}_{3}$ Transparent Ceramics," Optical Materials, Vol. 34, No. 8, 2012, pp. 1310-1313. doi:10.1016/j.optmat.2012.02.020

[25] K. Uchino, "Ferroelectric Devices," Marcel Dekker, New York, 2000.

[26] K. Uchino, "Electro-Optic Ceramics and Their Display Applications," Ceramics International, Vol. 21, No. 5, 1995, pp. 309-315. doi:10.1016/0272-8842(95)96202-Z

[27] A. Yariv and P. Yeh, "Optical Waves in Crystals: Propagation and Control of Laser Radiation," John Wiley \& Sons, New York, 1984. 